Frank Trommler (Hrsg.)

Amerika und die Deutschen. Die Beziehungen im 20. Jahrhundert 
Frank Trommler (Hrsg.)

\section{Amerika und die Deutschen}

Die Beziebungen im 20. Jabrhundert

Sonderausgabe

Springer Fachmedien Wiesbaden $\mathrm{GmbH}$ 


\section{CIP-Kurztitelaufnahme der Deutschen Bibliothek}

Sonderausgabe ausgewählter Beiträge aus:

Frank Trommler (Hrsg.), Amerika und die Deutschen.

Bestandsaufnahme einer 300jährigen Geschichte.

Alle Rechte vorbehalten

(C) 1986 Springer Fachmedien Wiesbaden

Ursprünglich erschienen bei Westdeutscher Verlag GmbH, Opladen 1986

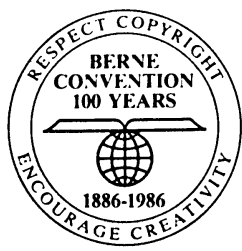

Das Werk einschließlich aller seiner Teile ist urheberrechtlich geschützt. Jede Verwertung außerhalb der engen Grenzen des Urheberrechtsgesetzes ist ohne Zustimmung des Verlags unzulässig und strafbar. Das gilt insbesondere für Vervielfältigungen, Übersetzungen, Mikroverfilmungen und die Einspeicherung und Verarbeitung in elektronischen Systemen.

Umschlaggestaltung: Horst Dieter Bürkle, Darmstadt

Satz: Satzstudio Frohberg, Freigericht 


\section{Vorbemerkung}

Der vorliegende Band ist eine im Buchhandel nicht erhältliche Sonderausgabe für die Bundeszentrale bzw. Landeszentralen für politische Bildung. Der Inhalt entspricht Teil 2 („Die Beziehungen im 20. Jahrhundert“) des Originalwerkes „Amerika und die Deutschen. Bestandsaufnahme einer 300jährigen Geschichte"; lediglich die beiden Beiträge, die dort das VIII. Kapitel bilden, konnten hier nicht aufgenommen werden. Das vollständige Inhaltsverzeichnis des Originalbandes findet sich am Schluß dieser Sonderausgabe.

Die Beiträge zu „Amerika und die Deutschen“ sind die überarbeiteten Vorträge, die am 3.-6. Oktober 1983 auf der, Tricentennial Conference of GermanAmerican History, Politics and Culture' an der University of Pennsylvania in Philadelphia gehalten wurden. Die Konferenz war Teil der Feiern zum 300jährigen Jubiläum der deutschen Einwanderung nach Nordamerika und wurde durch großzügige Unterstützung von Institutionen in den Vereinigten Staaten von Amerika und der Bundesrepublik Deutschland ermöglicht.

Während die meisten deutschen Teilnehmer selbst eine deutsche Version ihres auf englisch gehaltenen Vortrages anfertigten, gebührt Wolfgang Helbich das Verdienst, den Hauptteil der Konferenzbeiträge ins Deutsche übersetzt zu haben.

Westdeutscher Verlag 


\section{Inhalt}

Deutsch-amerikanische Beziehungen 1900-1950

Kaiserreich und Republik. Deutsch-amerikanische Beziehungen vor 1917

Reinhard R. Doerries . . . . . . . . . . . . . . . .

Die Vereinigten Staaten und die Weimarer Republik. Das Scheitern einer , besonderen Beziehung'

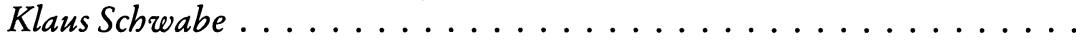

Franklin D. Roosevelt und die nationalsozialistische Bedrohung

der USA

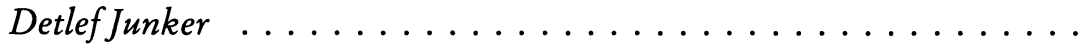

Von der Konfrontation zur Kooperation. Deutschland und die

Vereinigten Staaten 1933-1949

Gerbard L. Weinberg . . . . . . . . . . . . . . .

Produktion und Rehabilitation. Die wirtschaftlichen Grundlagen

der amerikanischen Förderung Westdeutschlands in der atlantischen

Gemeinschaft der Nachkriegszeit

Charles S. Maier . . . . . . . . . . . . . . . . .

Vom Nazismus zum NATOismus. Das westdeutsche

Wandlungswunder im Spiegel der Luce-Presse

Jost Hermand . . . . . . . . . . . . . . . . . . . . . . .

Das Bündnis: Eine Bestandsaufnahme 1983

Die deutsch-amerikanischen Beziehungen in den Nachkriegsjahrzehnten

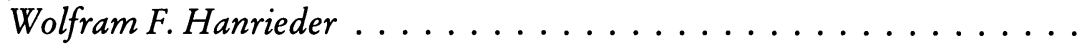

Der gegenwärtige Antiamerikanismus in der Bundesrepublik

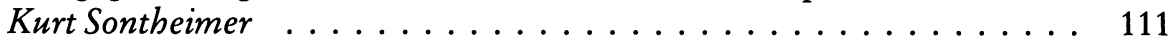

Mit Differenzen leben

Theo Sommer

Amerikanisch-deutsche Beziehungen. Ein Bündnis, das normal geworden ist

Fritz Stern 
Die Deutschamerikaner im 20. Jahrhundert

Affinität auf Widerruf. Amerikas willkommene und unwillkommene

Deutsche

Christine M. Totten

Die Rhetorik des Überlebens. Der Germanist in Amerika 1900-1925

Henry J. Schmidt

Erleichterte Amerikanisierung. Die Wirkung des Ersten Weltkriegs

auf die Deutschamerikaner in den zwanziger Jahren

La Vern J. Rippley

Ein problematisches Liebesverhältnis. Das amerikanische

Deutschlandbild seit 1930

Victor Lange . . . . . . . . . . . . . . . . . . . . . . . . .

\section{Einwanderung nach 1933}

Kontinuität im Wandel. Der deutsch-jüdische Einwanderer seit 1933

Herbert A. Strauss . . . . . . . . . . . . . . . . . . . . . . .

Kassandras mit deutschem Akzent

Anthony Heilbut . . . . . . . . . . . . . . . . . . . . . .

Weder Staat noch Synagoge. Der linke deutsch-jüdische emigrierte

Intellektuelle als repräsentativer Jude

Paul Breines

Die Kritische Theorie in den USA. Gedanken über vier Jahrzehnte ihrer Rezeption

Andrew Arato

Die literarische und akademische Abwanderung aus dem

Dritten Reich in die USA. Ein Forschungsbericht

John M. Spalek

Exkurs in die Psychoanalyse

Freuds Amerika

Peter Gay

\section{Amerikanismus und Massenkultur}

Massenkultur und Modernität. Notizen zu einer Sozialgeschichte des frühen amerikanischen und deutschen Films

Anton Kaes . . . . . . . . . . . . . . . . . . . . . . . . . . . . . . . . . . 261

Aufstieg und Fall des Amerikanismus in Deutschland

Frank Trommler

Die Autoren der Beiträge . . . . . . . . . . . . . . . . . 287

Inhaltsverzeichnis des Gesamtbandes . . . . . . . . . . . . . . 292 